\title{
Quantum theory of a resonant photonic crystal
}

\author{
Y. D. Chong,* David E. Pritchard, and Marin Soljačić \\ Department of Physics, Massachusetts Institute of Technology, Cambridge, Massachusetts 02139, USA
}

(Received 29 March 2007; published 27 June 2007)

\begin{abstract}
We present a quantum model of two-level atoms localized in a three-dimensional lattice based on the Hopfield polariton theory. In addition to a polaritonic gap at the excitation energy, a photonic band gap opens up at the Brillouin zone boundary. Upon tuning the lattice period or angle of incidence to match the photonic gap with the excitation energy, one obtains a combined polaritonic and photonic gap as a generalization of Rabi splitting. For typical experimental parameters, the size of the combined gap is on the order of $25 \mathrm{~cm}^{-1}$, up to $10^{5}$ times the detuned gap size. The dispersion curve contains a branch supporting slow-light modes with vanishing probability density of atomic excitations.
\end{abstract}

DOI: 10.1103/PhysRevB.75.235124

PACS number(s): 42.70.Qs, 32.80.Pj, 42.50.Fx

\section{INTRODUCTION}

Photonic crystals ( $\mathrm{PhCs}$ - _ systems in which the index of refraction varies periodically on the scale of light-are known to have an extraordinary ability to control the flow of light. ${ }^{1-3}$ While the underlying index of refraction in conventional $\mathrm{PhCs}$ is often taken to be the bulk value, interesting effects can occur when the underlying medium possesses resonances at wavelengths comparable to the lattice spacing; for instance, one can dramatically widen the photonic band gap by tuning the band gap frequency (e.g., by changing the lattice period) to match the resonance frequency. Such "resonant PhCs" can be realized using cold atoms in optical lattices, ${ }^{4-6} \mathrm{PhCs}$ made from polaritonic materials, ${ }^{7}$ and multiple-quantum-well arrays. ${ }^{8-10}$ Here, we will concentrate on the first class of resonant $\mathrm{PhCs}$, originally analyzed by Deutsch et al.,$^{5}$ who modeled the atoms in a one-dimensional (1D) optical lattice as a set of classical polarizable planes and showed that the interaction of the resonances with the periodicity of the system gives rise to a photonic band gap. Subsequently, van Coevorden et al. ${ }^{6}$ extended this study to three dimensions by solving Maxwell's equations in a lattice of resonating point dipoles using a $t$-matrix analysis.

In this paper, we present a simple three-dimensional (3D) quantum mechanical model of an atomic $\mathrm{PhC}$ in which the elementary excitations are polaritons: coherent superpositions of atomic excitations and photons. Several features of previous classical models appear naturally and with somewhat simpler interpretations in the quantum model. For instance, we show that the resonance-induced band gap arises as a generalization of Rabi splitting in a microcavity. Our model also exhibits the important "Bragg resonant" modes first studied in one dimension by Deutsch et al., who identified them with the standing electromagnetic wave that supports the optical lattice. ${ }^{5}$ Here, the Bragg resonant modes generalize to a family of modes occupying the boundary of the first Brillouin zone (BZ) and attached to the dispersion curve associated with atomic excitations; near the BZ boundary, they possess low group velocity but involve little excitation of the underlying medium, unlike states in "slowlight" systems. ${ }^{12}$

\section{MODEL HAMILTONIAN}

Consider $N$ localized two-level atoms of the same type in a fully filled 3D cubic lattice at sites $\vec{r}_{i}$ with lattice period $\ell$.
To facilitate calculation, we enclose the lattice in a periodic electromagnetic cavity of volume $V$, which reproduces the physical behavior inside a sufficiently large lattice. The Coulomb-gauge Hamiltonian is

$$
H=\sum_{i} \epsilon b_{i}^{\dagger} b_{i}+\sum_{\vec{k} \sigma} \hbar c|\vec{k}| a_{\vec{k} \sigma}^{\dagger} a_{\vec{k} \sigma}-\frac{e}{m c} \sum_{i} \vec{A}\left(\vec{r}_{i}\right) \cdot \vec{p}_{i},
$$

where $\epsilon$ is the energy difference between the atomic levels, $b_{i}^{\dagger} \equiv|1\rangle\left\langle\left. 0\right|_{i}\right.$ and $\left.b_{i} \equiv \mid 0\right\rangle\left\langle\left. 1\right|_{i}\right.$ are the level raising and lowering operators for atom $i$, and $a_{\vec{k} \sigma}^{\dagger}$ and $a_{\vec{k} \sigma}$ are creation and annihilation operators for photons with wave vector $\vec{k}$ and polarization $\sigma \cdot \vec{A}(\vec{r})$ is the vector potential,

$$
\vec{A}(\vec{r})=\sum_{\vec{k} \sigma} \sqrt{\frac{2 \pi \hbar c}{V|\vec{k}|}}\left(a_{\vec{k} \sigma} e^{i \vec{k} \cdot \vec{r}}+a_{\vec{k} \sigma}^{\dagger} e^{-i \vec{k} \cdot \vec{r}}\right) \hat{e_{k} \sigma}
$$

where $\hat{e}_{\vec{k} \sigma}$ is the unit polarization vector for $a_{\vec{k} \sigma}^{\dagger}$.

Let us suppose that the average number of atomic excitations in the system at any time is much less than $N$. In that case, the atomic excitations are approximately bosonic, in the same sense that spin waves are bosons. ${ }^{13}$ Therefore, the two photon polarizations, which excite orthogonal atomic states, decouple for each $k$. We thus drop the $\sigma$ label, with the understanding that the dispersion relations we will later obtain are doubly degenerate. This is also consistent with the weak polarization dependence obtained by van Coevorden et $a l .{ }^{6}$ In contrast, polarization effects play an important role in conventional $\mathrm{PhCs},{ }^{3}$ as well as multiple-quantum-well resonant $\mathrm{PhCs},{ }^{8-10}$ due to the finite size of the scattering centers.

We can treat the $\vec{r}_{i}$ in Eq. (1) as numbers (perfect lattice positions) rather than operators, since the electronic wave functions are typically much narrower than the lattice spacing. At each site, the momentum operator is

$$
\vec{p}_{i}=\frac{i}{\hbar} m \epsilon \vec{x}_{01}\left(b_{i}^{\dagger}-b_{i}\right), \quad \vec{x}_{01} \equiv\langle 1|\vec{x}| 0\rangle .
$$

Let us also define momentum-space excitation operators 


$$
b_{\vec{q}}=\frac{1}{\sqrt{N}} \sum_{i} e^{-i \vec{q} \cdot \vec{r}_{i}} b_{i}, \quad b_{\vec{q}}^{\dagger}=\frac{1}{\sqrt{N}} \sum_{i} e^{i \vec{q} \cdot \vec{r}_{i}} b_{i}^{\dagger},
$$

where the wave vectors $\vec{q}$ are restricted to the first BZ, corresponding to the fact that an excitation "wave" has no meaning between lattice points. As explained above, these are approximately bosonic: $\left[b_{\vec{q}}, b_{\vec{q}^{\prime}}^{\dagger}\right] \simeq \delta_{\vec{q} \vec{q}^{\prime}}$.

Substituting Eqs. (2)-(4) into Eq. (1), we obtain the microscopic polariton Hamiltonian first derived by Hopfield ${ }^{13}$ in the context of crystalline solids:

$$
\begin{aligned}
H= & \sum_{\vec{q}}\left(\epsilon b_{\vec{q}}^{\dagger} b_{\vec{q}}+\sum_{\vec{G}} \hbar c|\vec{q}+\vec{G}| a_{\vec{q}+\vec{G}}^{\dagger} a_{\vec{q}+\vec{G}}\right. \\
& -\sum_{\vec{G}} i C_{\vec{q}+\vec{G}}\left\{\left(b_{\vec{q}}^{\dagger} a_{\vec{q}+\vec{G}}-a_{\vec{q}+\vec{G}}^{\dagger} b_{\vec{q}}\right)\right. \\
& \left.\left.+\left[b_{\vec{q}}^{\dagger} a_{-(\vec{q}+\vec{G})}^{\dagger}-a_{-(\vec{q}+\vec{G})} b_{\vec{q}}\right]\right\}\right),
\end{aligned}
$$

where $\vec{G}$ run over all reciprocal lattice vectors, and

$$
C_{\vec{q}+\vec{G}}=\sqrt{\frac{2 \pi \alpha N}{|\vec{q}+\vec{G}| V}} \epsilon x_{01},
$$

where $\alpha$ is the fine structure constant. The atom-photon interaction consists of two parts. The first part, on the second line of Eq. (5), describes the lattice absorbing a photon with wave vector $\vec{q}+\vec{G}$ to create an atomic excitation with wave vector $\vec{q}$ and the reverse process of destroying an excitation to emit a photon. The remaining interaction terms describe the creation and annihilation of associated pairs of photons and atomic excitations. The usual way to diagonalize Eq. (5) is to introduce polariton operators $\alpha_{\vec{q}}$ (Refs. 13 and 14) for each reduced wave vector $\vec{q}$ as linear combinations of $b_{\vec{q}}$, $b_{-\vec{q}}^{\dagger}, a_{\vec{q}+\vec{G}}$, and $a_{-\vec{q}+\vec{G}}^{\dagger}$ (for all $\vec{G}$ ). Stipulating that these act as decoupled lowering operators for $H$, one obtains the polariton energies as solutions of a $(2 n+1) \times(2 n+1)$ eigenvalue problem for each $\vec{q}$, where $n$ is the number of BZs included in the calculation. Higher BZs were first included into the Hopfield theory by Knoester and Mukamel ${ }^{14}$ in their calculation of polariton-mediated intermolecular forces in solids. There, the photons in the higher BZs were taken to be decoupled from the atomic excitations, which was appropriate since the BZ energy was many orders of magnitude larger than $\epsilon$. In our system, the two energies are comparable, and we must incorporate the interaction up to at least the secondorder zones.

It simplifies the calculations to drop the "counter-rotating" interaction terms in Eq. (5) describing the creation and annihilation of pairs. This is physically justifiable even though the discarded terms have the same coupling strength $C_{\vec{q}+\vec{G}}$ as the remaining interaction terms, because the pair creation and annihilation process is a quantum mechanical fluctuation of the "vacuum" with a finite energy gap $\epsilon+\hbar c|\vec{q}|$. For $\epsilon$ and $\hbar c|\vec{q}|$, both on the order of $\mathrm{eV}$, and lattice periods at optical wavelengths, $C_{\vec{q}} \sim 10^{-4} \mathrm{eV} \ll \epsilon+\hbar c|\vec{q}|$. Such fluctuations are thus extremely rare and have a negligible effect on particle energies. The interaction terms describing the conversion of

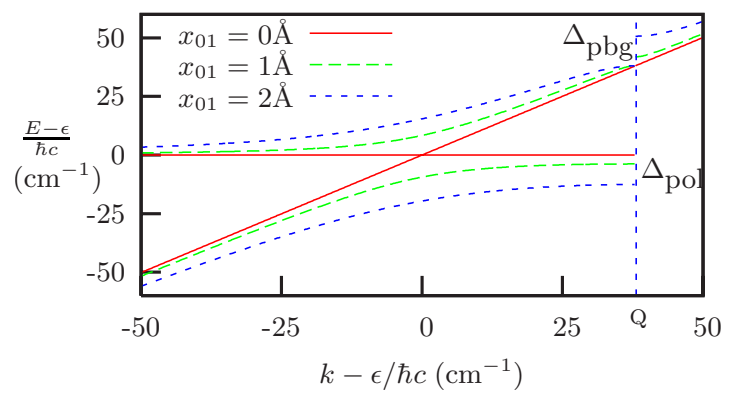

FIG. 1. (Color online) Single-polariton dispersion for a 3D cubic lattice along [100] in the extended zone scheme, with $\epsilon=3 \mathrm{eV}$ and three different coupling strengths, associated with the parameters $x_{01}=0$ (noninteracting), $1 \AA\left(C_{Q} \sim 0.18 \mathrm{meV}\right)$, and $2 \AA\left(C_{Q}\right.$ $\sim 0.35 \mathrm{meV})$. The vertical dashed line indicates the BZ boundary at $|\vec{Q}|=1.00025 \epsilon / \hbar c$. The graphs are generated numerically from Eq. (7), summing over 125 BZs.

a real photon into an atomic excitation, and vice versa, remain important: Since the existing particle possesses energy, these processes involve a much smaller energy fluctuation. The approximation holds provided we look at values of $|\vec{q}|$ comparable to both $\epsilon / \hbar c$ and the BZ energy, which is exactly the regime we are interested in.

The Hamiltonian now decouples into $N$ independent pieces, $H=\sum_{\vec{q}} H_{\vec{q}}$, one for each reduced wave vector:

$$
\begin{aligned}
H_{\vec{q}}= & \epsilon b_{\vec{q}}^{\dagger} b_{\vec{q}}+\sum_{\vec{G}} \hbar c|\vec{q}+\vec{G}| a_{\vec{q}+\vec{G}}^{\dagger} a_{\vec{q}+\vec{G}} \\
& -\sum_{\vec{G}} i C_{\vec{q}+\vec{G}}\left(b_{\vec{q}}^{\dagger} a_{\vec{q}+\vec{G}}-a_{\vec{q}+\vec{G}}^{\dagger} b_{\vec{q}}\right) .
\end{aligned}
$$

This says that each photon mixes with all other photons having wave vectors that differ by a reciprocal lattice vector, as one expects of a PhC system. Here, the mixing is mediated by the atom-photon interaction. Since Eq. (7) has the quadratic form $\Sigma_{i j} \beta_{i}^{\dagger} \mathcal{H}_{i j} \beta_{j}$, it can be diagonalized as $\Sigma_{n} E_{n} \alpha_{n}^{\dagger} \alpha_{n}$, where the $\alpha$ are boson operators defined by $\alpha_{n}=\sum_{j} w_{j}^{* n} \beta_{j}, E_{n}$ is the $n$th eigenvalue of $\mathcal{H}$, and $w^{n}$ is the corresponding eigenvector. We can thus obtain the polariton energies $E_{\vec{q}}^{n}$ by including a finite number of BZs in the sum and diagonalizing the associated matrix.

\section{BAND STRUCTURE}

Figure 1 shows the polariton dispersion curves along the [100] direction for a blue-detuned optical lattice. The interaction opens up two energy gaps in the polariton spectrum: an indirect "polaritonic gap" $\Delta_{\text {pol }}$ at $\epsilon$ due to the repulsion between the bare dispersion curves and a photonic band gap $\Delta_{\text {pbg }}$ at $\hbar c|\vec{Q}|$, where $\vec{Q}$ is the BZ boundary. We have also calculated the density of polariton states; after integrating over all angles, we find that the density of states is enhanced near the band edges but remains nonzero at all energies because the exact sizes and positions of the gaps vary with angle. The system therefore does not possess a complete gap, essentially because of the weakness of the electromagnetic 


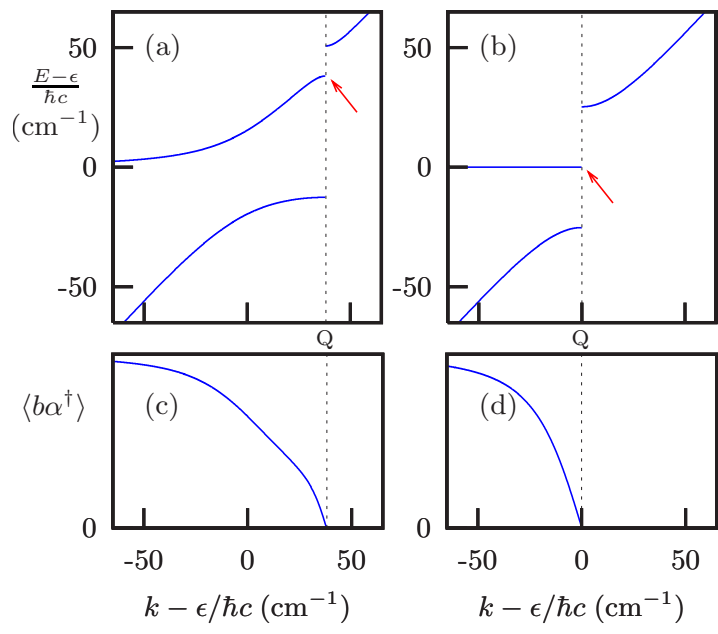

FIG. 2. (Color online) Single-polariton dispersion along [100], with $\epsilon=3 \mathrm{eV}, x_{01}=2 \AA$, and different lattice periods: (a) $|\vec{Q}|$ $=1.00025 \epsilon / \hbar c$ and (b) $|\vec{Q}|=\epsilon / \hbar c$. Plots (c) and (d) show the corresponding overlaps of the polariton with the bare excitation, $\left\langle 0\left|b_{\vec{q}} \alpha_{\vec{q}}^{\dagger}\right| 0\right\rangle$, for the polaritons on the dispersion curve leading to the purely photonic state at $\vec{q}=\vec{Q}$ [indicated with arrows in (a) and (b)], which have no atomic component.

interaction. The gap sizes vary continuously as we change the lattice period $a$, and thus $V$ (keeping $N$ and all other parameters constant). As shown in Fig. 2, the gaps meet and become significantly enhanced when the BZ boundary intersects the crossing point of the bare dispersion curves.

To understand the nature of the spectrum at the $\mathrm{BZ}$ boundary, consider a photon with wave vector $\vec{k}=\vec{Q}$ along one of the faces of the cube. There is another such photon, with wave vector $\vec{Q}+\vec{G}^{\prime}$ lying on the opposite face, such that $|\vec{Q}|=\left|\vec{Q}+\vec{G}^{\prime}\right|$. (When $\vec{Q}$ lies on an edge or corner of the BZ boundary, there are more partners; we will not consider these cases, but they can be treated in a similar fashion.) The two photons mix strongly since they have the same energy, so we can neglect the other photon states and use the effective Hamiltonian

$$
\widetilde{H}_{\vec{Q}}=\left[\begin{array}{c}
b_{\vec{Q}} \\
a_{\vec{Q}} \\
a_{\vec{Q}+\vec{G}^{\prime}}
\end{array}\right]+\left[\begin{array}{ccc}
\epsilon & -i C_{Q} & -i C_{\vec{Q}} \\
i C_{\vec{Q}} & \hbar c|\vec{Q}| & 0 \\
i C_{\vec{Q}} & 0 & \hbar c|\vec{Q}|
\end{array}\right]\left[\begin{array}{c}
b_{Q} \\
a_{\vec{Q}} \\
a_{\vec{Q}+\vec{G}^{\prime}}
\end{array}\right] .
$$

Thus, the polariton energies at the BZ boundary are

$$
\begin{gathered}
E_{\vec{Q}}^{0}=\hbar c|\vec{Q}|, \\
E_{\vec{Q}}^{ \pm}=\frac{\epsilon+\hbar c|\vec{Q}|}{2} \pm \sqrt{\left(\frac{\epsilon-\hbar c|\vec{Q}|}{2}\right)^{2}+2 C_{\vec{Q}}^{2}} .
\end{gathered}
$$

These are exactly the energy levels resulting from Rabi splitting of a two-level atom interacting with two counterpropagating photon states with wave vectors $\pm \vec{Q}$, with an effective cavity size $V / N$. In the exactly tuned case $\epsilon=\hbar c|\vec{Q}|, E_{Q}^{ \pm}$has a special significance: As shown in Fig. 2(b), these are the upper and lower edges of the band gap. The resonant enhancement of the band gap in this system is thus a manifestation of the Purcell effect. ${ }^{15}$ Intuitively, we can imagine enclosing a single atom in a microcavity with the dimensions of the unit cell; if the cavity walls are mirrors, the atom sees a lattice of atoms similar to the one considered here.

We have checked Eq. (9) against numerical solutions of Eq. (7) including the 125 lowest BZs for various values of $\vec{Q}$ along the BZ boundary up to $40^{\circ}$ from the [100] direction. For $\epsilon=3 \mathrm{eV}$ and $x_{01}=2 \AA$, the error is always less than $0.02 \mathrm{~cm}^{-1}, 3$ orders of magnitude smaller than the maximum gap size.

The size of the gaps in the exactly tuned limit can be estimated by substituting $\epsilon=\hbar c\left|\vec{Q}^{\prime}\right|$ into Eq. (9):

$$
\Delta \approx \sqrt{2} C_{\epsilon / \hbar c}^{\prime}=\sqrt{\frac{4 \alpha x_{01}^{2} \epsilon^{4}}{\pi^{2}(\hbar c)^{2}}} .
$$

For $\epsilon, \quad \hbar c|\vec{Q}| \approx 3 \mathrm{eV}$ and $x_{01} \approx 2 \AA, \quad \Delta / \hbar c \approx 25 \mathrm{~cm}^{-1}$ $\left(\sim 10^{-4} \epsilon\right)$, in agreement with Fig. 2(b). We can also obtain limiting expressions for the gaps when they are significantly decoupled. Consider $|\vec{Q}|>\epsilon / \hbar c$, as in Fig. 2(a). Away from the BZ boundary, we can neglect the effect of photons in higher BZs, and the effective Hamiltonian matrix is $\mathcal{H}=\left[\epsilon,-i C_{\vec{q}} ; i C_{\vec{q}}, \hbar c|\vec{q}|\right]$, with eigenvalues

$$
E_{\vec{q}}^{ \pm}=\frac{\epsilon+\hbar c|\vec{q}|}{2} \pm \sqrt{\left(\frac{\epsilon-\hbar c|\vec{q}|}{2}\right)^{2}+C_{\vec{q}}^{2}}
$$

The contribution to the indirect polaritonic gap from the large- $q$ branch of the dispersion curve, which is truncated at the BZ boundary, is obtained from the large- $q$ expansion of Eq. (11) evaluated at $\vec{q}=\vec{Q}$. The contribution from the small$q$ branch cannot be found by setting $\vec{q}=0$ in Eq. (11) due to our preceding approximations, so we instead calculate an upper bound on it by evaluating it at the minimum, $|\vec{q}|$ $=\epsilon / 2 \hbar c$. The resulting polaritonic gap is

$$
\Delta_{\mathrm{pol}}^{\prime} \simeq \frac{4 C_{\epsilon / \hbar c}^{2}}{\epsilon}+\frac{C_{\vec{Q}}^{2}}{\hbar c Q} .
$$

With the same lattice parameters, $\Delta_{\text {pol }}^{\prime} \approx 10^{-3} \mathrm{~cm}^{-1}$ $\left(\sim 10^{-8} \epsilon\right)$. From the large- $|\vec{Q}|$ expansion of Eq. (9), the photonic band gap is $\Delta_{\mathrm{pbg}}^{\prime}=C_{\vec{Q}}^{2} / \hbar c|\vec{Q}|$, strictly smaller than Eq. (12). Therefore, the effects of the polaritonic interaction are very small when the system is detuned.

This model can also be used to study the quasi-1D geometry considered by many authors, in which atoms are trapped along periodically stacked infinite sheets. Consider a 3D lattice in which the lattice spacing in one of the directions, $\ell_{1}$, is much larger than the spacing in the other two directions. The relevant wave vectors, lying on the BZ boundaries closest to the origin, have magnitude $\left|\vec{Q}_{1}\right|=\pi / \ell_{1}$ and point in the direction of stacking. In this regime, this model can be directly compared with the semiclassical analysis of Deutsch 
et al. ${ }^{5}$ For instance, the semiclassical theory predicts band gaps from $E_{-}^{(c l)}$ to $\epsilon$ and from $\hbar c\left|\vec{Q}_{1}\right|$ to $E_{+}^{(c l)}$ for blue-detuned lattices. A short calculation, using Eqs. (15)-(19) of that paper, yields

$$
E_{ \pm}^{(c l)} \approx \frac{\epsilon+\hbar c|\vec{Q}|}{2} \pm \sqrt{\left(\frac{\epsilon-\hbar c|\vec{Q}|}{2}\right)^{2}+2 \frac{3 \hbar^{2} c \gamma \eta}{2\left|\vec{Q}_{1}\right|}}
$$

where $\eta$ is the surface density along each sheet and $\gamma \ll\left(E_{ \pm}-\epsilon\right) / \hbar$ is the linewidth of the atomic transition. Using the golden rule prescription for the natural linewidth, ${ }^{11}$ $\gamma=\left(4 \alpha \epsilon^{3} x_{01}^{2}\right) /\left(3 \hbar^{3} c^{2}\right)$, this reduces to Eq. (9) with $C_{Q}^{2}$ replaced by $C_{Q}^{2} \cdot \epsilon / \hbar c\left|\vec{Q}_{1}\right|$. The band gaps predicted by the semiclassical and quantum mechanical theories are thus similar for $\epsilon \sim \hbar c\left|\vec{Q}_{1}\right|$, which is also the regime where the band gaps are significant. In the exactly tuned case, the results are identical, and one obtains

$$
\Delta_{1 d}=2 \epsilon x_{01} \sqrt{\alpha \eta} .
$$

Actual 1D and/or two-dimensional (2D) lattices are more problematic since each atomic excitation is coupled to photons with a continuum of wave numbers in the transverse direction, which smears out the gaps. One might avoid this using an actual cavity in the transverse direction, making the electromagnetic field effectively $1 \mathrm{D}$ and/or $2 \mathrm{D}$.

\section{SLOW POLARITON MODES}

The energy $E_{Q}^{0}$ in Eq. (9) corresponds to a polariton created by the operator $\left(a_{Q}^{\dagger}-a_{Q+G^{\prime}}^{\dagger}\right) / \sqrt{2}$. This remains an exact polariton state when we include higher BZs in the effective Hamiltonian. (In fact, there is a family of such states for each pair of BZ boundaries.) These "purely photonic" polaritons are reminiscent of "dark states" in electromagnetically induced transparency (EIT), ${ }^{12}$ since Eq. (8) is identical to the EIT effective Hamiltonian with the atomic excitation and two photon modes acting as the levels of the $\Lambda$ system. In EIT, a dark state arises: a coherent superposition of atomic levels that does not couple to the radiation. The analog in our case is a noninteracting photonic state, with no atomic component. Its classical limit is a standing electromagnetic wave commensurate with the lattice. Since the laser light that supports the lattice always falls exactly on the BZ boundary, ${ }^{5}$ the stability of the optical lattice relies on the existence of such standing wave modes; other modes are Bragg reflected away. In a sense, the lattice "selects" the standing wave modes from the incoming laser light. Similar modes have been observed in other resonant $\mathrm{PhC}$ systems. ${ }^{8-10}$ We have shown here that in the self-consistent limit of complete quantum coherence and low excitation density, this selection takes place at the quantum state level. Only the purely photonic polaritons can support a macroscopic population, since they are the only elementary excitations of the interacting system with zero atomic component.

In the 3D system, there is a family of purely photonic polaritons everywhere on the boundary of the first BZ. Remarkably, these states are attached to the slow, "atomic"

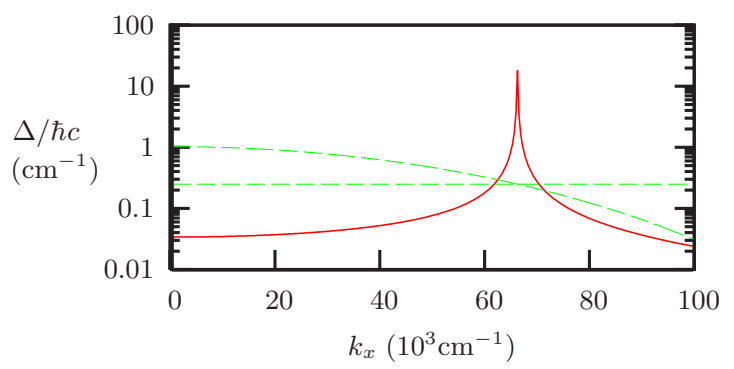

FIG. 3. (Color online) Photonic gap at wave vectors $\vec{Q}=\left[k_{x}, \pi / \ell, 0\right]$ along the BZ boundary for $\epsilon=3 \mathrm{eV}, x_{01}=2 \AA$, and $\pi / \ell=0.9 \epsilon / \hbar c=1.4 \times 10^{5} \mathrm{~cm}^{-1}$ (red detuned). The dashed lines show $k_{y}$ vs $k_{x}$ for the surface $|\vec{k}|=\epsilon / \hbar c$ and the BZ boundary; here, the ordinate is not drawn to scale. The gap is largest at the intersection of the two surfaces, i.e., $|\vec{Q}|=\epsilon / \hbar c$.

branch of the dispersion curve. These appear to be analogs of the slow, nondegenerate, longitudinal electromagnetic modes that appear in the classical $t$-matrix calculation of Coevorden et al. ${ }^{6}$ Our model shows that the photonic component along this branch goes continuously from nearly zero to unity as we approach the BZ boundary, as shown in Figs. 2(c) and 2(d). Therefore, by exciting polaritons over the range

$$
\left|\frac{\epsilon-\hbar c|\vec{Q}|}{\hbar c}\right| \sim \frac{C_{\vec{Q}}}{\hbar c} \sim 10 \mathrm{~cm}^{-1}
$$

around wave vector $\vec{Q}$, one could create a wave packet that propagates slowly but has low atomic excitation density.

\section{CONCLUSION}

We have presented a quantum model for an atomic lattice that applies directly to optical lattices filled with cold atoms, containing behavior similar to other resonant $\mathrm{PhC}$ systems. The system possesses two gaps (polaritonic and photonic) at each angle and can be tuned so that the gaps meet to create a combined gap orders of magnitude larger than the individual detuned gaps, in a process analogous to microcavity Rabi splitting; however, there does not exist a complete gap. The quantum analysis yields a branch of the dispersion curve that has low group velocity and atomic component vanishing at the BZ boundary.

These effects could be explored with alkali atoms held in a cubic lattice made by near-IR light, by introducing a probe beam at an angle to the axis of the lattice. One should choose an atomic transition $\epsilon$ such that $1 \leqslant \epsilon \ell / \pi \hbar c \leqslant \sqrt{3}$, where $\ell$ is the lattice period, and use probe wave vectors with magnitude lying in the range $\Delta / \hbar c \sim 10 \mathrm{~cm}^{-1}$ around $|\vec{q}|=\epsilon / \hbar c$, at an angle $\cos ^{-1}(\pi \hbar c / \epsilon \ell)$ to a lattice axis (Fig. 3). Although the present theory applies to an infinite lattice, the predicted frequency shifts may be observable close to the atomic resonance, even in a lattice of about 100 atoms on a side.

We have treated the atomic positions as fixed, as would be the case for a strongly confining optical lattice where the rate at which each atom tunnels to a different lattice site is negligible compared to the radiative lifetime. The presence of 
nonzero hopping amplitudes would add an imaginary part to the polariton energies, proportional to the tunneling rate. The size of the band gaps would be reduced by the corresponding amount.

Finally, it is interesting to note that the gap in Eq. (10), which scales as $\epsilon$ relative to the photon energy, is $O\left(10^{-2}\right) \epsilon$ for $\mathrm{x}$ rays. Aspects of this theory might thus be applicable to crystalline solids in the $\mathrm{x}$-ray regime, where a similar effect-super-radiant scattering enhancement due to nuclear resonances-is known to exist. ${ }^{16}$

\section{ACKNOWLEDGMENTS}

We would like to thank R. W. Boyd, J. D. Joannopoulos, V. Vuletic, A. Gordon, and F. X. Kaertner for helpful discussions. *cyd@mit.edu

${ }^{1}$ S. John, Phys. Rev. Lett. 58, 2486 (1987).

${ }^{2}$ E. Yablonovitch, Phys. Rev. Lett. 58, 2059 (1987).

${ }^{3}$ J. D. Joannopoulos, R. D. Meade, and J. N. Winn, Photonic Crystals: Molding the Flow of Light (Princeton University Press, Princeton, NJ, 1995).

${ }^{4}$ P. S. Jessen and I. H. Deutsch, in Advances in Atomic, Molecular, and Optical Physics, edited by B. Bederson and H. Walther (Academic, Cambridge, 1996), Vol. 37.

${ }^{5}$ I. H. Deutsch, R. J. C. Spreeuw, S. L. Rolston, and W. D. Phillips, Phys. Rev. A 52, 1394 (1995).

${ }^{6}$ D. V. van Coevorden, R. Sprik, A. Tip, and A. Lagendijk, Phys. Rev. Lett. 77, 2412 (1996).
${ }^{7}$ K. C. Huang, P. Bienstman, J. D. Joannopoulos, K. A. Nelson, and S. Fan, Phys. Rev. Lett. 90, 196402 (2003).

${ }^{8}$ L. C. Andreani, Phys. Status Solidi B 188, 29 (1995).

${ }^{9}$ M. Hübner, J. P. Prineas, C. Ell, P. Brick, E. S. Lee, G. Khitrova, H. M. Gibbs, and S. W. Koch, Phys. Rev. Lett. 83, 2841 (1999). ${ }^{10}$ L. I. Deych and A. A. Lisyansky, Phys. Rev. B 62, 4242 (2000).

${ }^{11}$ E. Fermi, Rev. Mod. Phys. 4, 87 (1932).

${ }^{12}$ S. E. Harris, Phys. Today 50(7), 36 (1997).

${ }^{13}$ J. J. Hopfield, Phys. Rev. 112, 1555 (1958).

${ }^{14}$ J. Knoester and S. Mukamel, Phys. Rev. A 40, 7065 (1989).

${ }^{15}$ E. M. Purcell, Phys. Rev. 69, 681 (1946).

${ }^{16}$ G. V. Smirnov, Hyperfine Interact. 123-124, 31 (1999). 